\title{
Internal Lifschitz Singularities for One Dimensional Schrödinger Operators
}

\author{
G. A. Mezincescu ${ }^{\star}$ \\ Institut für Mathematik, Ruhr-Universität Bochum, Germany
}

Received: 19 June 1992/in revised form: 6 May 1993

Abstract. The integrated density of states of the periodic plus random one-dimensional Schrödinger operator $H_{\omega}=-\Delta+V_{\text {per }}+\sum_{i} q_{i}(\omega) f(0-i) ; f \geq 0$, $q_{i}(\omega) \geq 0$, has Lifschitz singularities at the edges of the gaps in $S p\left(H_{\omega}\right)$. We use Dirichlet-Neumann bracketing based on a specifically one-dimensional construction of bracketing operators without eigenvalues in a given gap of the periodic ones.

\section{Introduction}

In this paper we will consider the behavior of the integrated density of states (IDS) for the one-dimensional random Schrödinger operator.

$$
\begin{aligned}
H_{\omega}(g) & =-\Delta+V_{\mathrm{per}}+g V_{\omega} \\
& =T+g V_{\omega},
\end{aligned}
$$

where

$$
V_{\text {per }}(x+1)=V_{\text {per }}(x)
$$

is a periodic, piecewise continuous function, $g>0$,

$$
V_{\omega}(x)=\sum_{n \in \mathbb{Z}} q_{n}(\omega) f(x-n)
$$

with piecewise continuous $f \geq 0$, supp $f \subset\left(-\frac{1}{2}, \frac{1}{2}\right)$, and $q_{n}(\omega)$ are independent, identically distributed (iid) random variables. Their distribution function $\mu$ is assumed to have compact support

$$
\operatorname{supp} \mu \subset[0,1]
$$

\footnotetext{
* Present and permanent address: Institutul de Fizica şi Tehnologia Materialelor, C.P. MG-7, R-76900 Bucureşti, Măgurele, România
} 
and

$$
\begin{aligned}
\mu[0, x) & =O\left(x^{\delta_{-}}\right), \\
\mu(1-x, 1] & =O\left(x^{\delta_{+}}\right)
\end{aligned}
$$

for some $\delta_{-}, \delta_{+} \geq 0$.

The integrated density of states is defined by

$$
\sigma\left(E, H_{\omega}\right)=\sigma(E)=\lim _{L \uparrow \infty} \frac{1}{\left|\Lambda_{L}\right|} \#\left(H_{\omega, \Lambda_{L}}^{b, c}-E\right),
$$

where $\Lambda_{L}=(-L, L), H_{\omega, \Lambda_{L}}^{b, c}$ is some restriction defined by boundary conditions (b.c.) of $H_{\omega}$ to $L^{2}\left(\Lambda_{L}\right)$ and $\#(H)$ is the number of eigenvalues of the operator $H$ which are $\leq 0$. The limit in Eq. (1.6) exists under much weaker assumptions on the stochastic potential $V_{\omega}$ implied by than those in Eq. (3)-(5) [15].

An intuitive physical argument led I. M. Lifschitz to predict that the density of states, $\varrho(E)=d \sigma / d E$, has an universal type asymptotic behavior

$$
\ln \varrho(E) \sim-\text { const }\left|E-E_{c}\right|^{-\frac{d}{2}},
$$

for $E \in S p H_{\omega}$ near the fluctuative spectral edges $E_{c}$ of $H_{\omega}$ (here $d$ is the space dimension; $d=1$ in this paper). There are many rigorous proofs of somewhat weaker statements,

$$
\lim _{S p\left(H_{\omega}\right) \ni E \rightarrow E_{c}} \frac{\ln |\ln | \sigma E)-\sigma\left(E_{c}\right)||}{\ln \left|E-E_{c}\right|}=-\frac{d}{2},
$$

or

$$
\ln \left|\sigma(E)-\sigma\left(E_{c}\right)\right|=-\frac{\Phi_{c}(E)}{\left|E-E_{c}\right|^{d / 2}},
$$

with $\Phi_{c}$ having sometimes a weak singularity $\left(\sim \ln \left|E-E_{c}\right|\right)$ at $E_{c}[1,3,5,7,9-14$, 17-19]. But nearly all have dealt only with the lowest spectral edges of $H_{\omega}$.

For the finite-difference analogue of $H_{\omega}$, a theorem of type Eq. (1.9) was proven [11] for all spectral edges, while a simpler proof of Eq. (1.8) may be found in [18]. Kirsch and Nitzschner [6] have considered a disordered one-dimensional KronigPenney model (with point interactions) which has an infinite number of gaps in its spectrum [4]. The upper spectral edges (lowest edges of the gaps) in this model are nonfluctuative and $\lim _{S p\left(H_{\omega}\right) \ni E \rightarrow E_{c}} \frac{\ln \left|\sigma(E)-\sigma\left(E_{c}^{-}\right)\right|}{\ln \left|E-E_{c}\right|}=\frac{1}{2}$. Near one-half of the fluctuative spectral edges they have proven that

$$
\lim _{S p H_{\omega} \ni E \rightarrow E_{c}} \sup \frac{\ln |\ln | \sigma(E)-\sigma\left(E_{c}\right)||}{\ln \left|E-E_{c}\right|} \leq-\frac{1}{2} .
$$

The model considered in [6] has an infinite number of gaps for any (positive) value of the coupling constant $g$ due to the zero-range potential. In our case, since for $g=0$ the one-dimensional Schrödinger operator with periodic potential $T$ has generically an infinite number of gaps, it seems reasonable to assume that, for sufficiently small $g, H_{\omega}$ will have the same property. Indeed, Kirsch and Martinelli [4] have proved:

Theorem 1. Let $H_{\omega}$ be given by Eqs. (1)-(5) and

$$
T(g)=T+g \sum_{n \in \mathbb{Z}} f(x-n) .
$$


Then, the set

$$
\{x \in \mathbb{R} \mid x \notin S p T(0) \cup S p T(g), \sigma(x ; T(0))=\sigma(x ; T(g))\} \in \operatorname{Res}\left(H_{\omega}\right) .
$$

Proof. By ergodicity $S p H_{\omega}(g)$ is a.s. nonrandom and if $x \in S p\left(H_{\omega}\right)$ then $x$ is a growth point for $\sigma\left(x ; H_{\omega}\right)$. But

$$
T=T(0) \leq H_{\omega}(g) \leq T(g)
$$

so that

$$
\sigma(E ; T(g)) \leq \sigma\left(E ; H_{\omega}(g)\right) \leq \sigma(E ; T(0)) .
$$

Remark 1. If $\operatorname{supp} \mu=[0,1]$, then Eq. (1.12) yields all the gaps in $S p\left(H_{\omega}\right)$ [4].

Since the spectral edges of $T(g)$ are, for small enough $g$, analytic functions of $g$, it is obvious that for small enough $g, g<g_{n}^{c}$, the $n^{\text {th }}$ gap ${ }^{1}$ of $T=T(0)$ is not closed. In the following we shall assume that $g<g_{n}^{c}$ for the particular gap we are studying and, by redefining $g_{n}^{c} f=f$, we may assume $g \leq 1$.

Let $T_{1}^{\zeta}(g), \zeta=e^{i \theta} \in U(1)$ be the quasiperiodic restriction of $T(g)$ to $L^{2}(0,1)$, $\mathscr{D}\left(T_{1}^{\zeta}\right)=\left\{\varphi \in C^{1}[0,1] \mid \varphi^{\prime \prime} \in L^{2}, \varphi(1)=\zeta \varphi(0), \varphi^{\prime}(1)=\zeta \varphi^{\prime}(0)\right\}$. Let $\lambda_{n}(\theta, g)=$ $\lambda_{n}\left[T_{1}^{\zeta}(g)\right]$ be its $n^{\text {th }}$ eigenvalue (in nondecreasing order).

Define

$$
E_{n-1}^{+}=\lambda_{n}((n-1) \pi, 0) ; \quad E_{n}^{-}=\lambda_{n}(n \pi, 1), \quad n=1,2, \ldots
$$

By Theorem (1.1) and Theorem XIII.90 of [16] the set

$$
\mathscr{E}=\left\{E_{0}^{+}\right\} \bigcup_{\substack{n=1 \\ E_{n}^{-}<E_{n}^{+}}}\left\{E_{n}^{-}, E_{n}^{+}\right\} \subset S p\left[H_{\omega}\right],
$$

is a set of finite spectral edges of $H_{\omega}$, and, by the previous Remark, if supp $\mu=[0,1]$ there are no other (finite) edges.

Now we can state our main result:

Theorem 2. Let $H_{\omega}$ be given by Eqs. (1.1)-(1.3) with $\mu$ satisfying (1.4) and (1.5) Then, for any edge $E^{c} \in \mathscr{E}$ :

$$
\lim _{S p\left(H_{\omega}\right) \ni E \rightarrow E_{c}} \frac{\ln |\ln | \sigma(E)-\sigma\left(E_{c}\right)||}{\ln \left|E-E_{c}\right|}=-\frac{1}{2} .
$$

Remark2. Inspection of the proof will show that the result may be extended to $f$ with larger support than $[0,1]$ but with $f \geq 0$. In particular the result of Kirsch and Simon [7] for $E_{0}^{+}$: the limit in Eq. (1.18) is equal to $-1 / \min (\alpha, 2)$, if $f=O\left(|x|^{-\alpha-1}\right)$, $\alpha>0$, as $|x| \rightarrow \infty$, extends to all $\mathscr{E}$.

Thus, the result known for the lowest edge [7] is valid for all the other edges. We will prove Theorem 2 by a combination of standard techniques: Dirichlet-Neumann bracketing and large deviation estimates [5, 6, 10-12, 17-19].

The bracketing operators for an arbitrary partition have eigenvalues inside the gaps of $S p\left(H_{\omega}\right)$. The one-dimensional case discussed in this paper is distinguished by the fact that the bracketing restrictions of the periodic operators $T(g)$ to an interval have exactly one eigenvalue in each of the gaps of $S p[T(g)]$. This is the content

\footnotetext{
1 Generically $S p(T(g))$ has an infinite number of gaps. Only for some rather special $V_{\text {per }}$ (elliptic functions) there is a finite number
} 
of Theorem 3 in the next section, ${ }^{2}$ which also contains some known facts on onedimensional Schrödinger operators with various boundary conditions on an interval. In Sect. 3 we will show that, by an adequate choice of the partition, the eigenvalues of the bracketing operators in a given gap may be pushed to a predetermined edge. Using these operators, the proof of Theorem 2 becomes a rather standard undertaking and will be sketched in the last section.

\section{Some Facts on $-\Delta+V$ on an Interval}

Let the real function $V$ be piecewise continuous one some finite interval $J=[a, b]$ and define the operator $T_{J}$ by

$$
\left(T_{J}^{\text {b.c. }} f\right)(x)=-\frac{d^{2} f}{d x^{2}}+V(x) f(x)
$$

on $L^{2}(J)$ with

$$
\mathscr{D}\left(T_{y}^{\text {b.c. }}\right)=\left\{f \in C^{1}(J) \mid f^{\prime \prime} \in L^{2}(J), f \text { satisfies boundary conditions }\right\} .
$$

We will consider the following types of boundary conditions which lead to selfadjoint operators bounded from below and having compact resolvents:
a) N-Neumann: $f^{\prime}(a)=f^{\prime}(b)=0$;
b) D - Dirichlet: $f(a)=f(b)=0$;
c) $\zeta$-quasiperiodic: $f^{\prime}(b)=\zeta f(a), f^{\prime}(b)=\zeta f^{\prime}(a), \zeta \in U(1)$.

Whenever it is unambiguous we will write $T^{\text {b.c. }}$ for $T_{J}^{\text {b.c. }}$. The following proposition summarizes known facts on the eigenvalues and eigenfunctions of $T^{\text {b.c. }}[2,16]$.

Proposition 1. Let $T^{\text {b.c. }}$ be defined as above, $\varepsilon_{n}^{N}, \varepsilon_{n}^{D}, \varepsilon_{n}(\zeta), n=1,2, \ldots$, be their eigenvalues arranged in a nondecreasing sequence and $u_{n}^{\text {b.c. }}$ - the corresponding eigenfunctions. Then

1) $\varepsilon_{n}^{N}, \varepsilon_{n}^{D}$ and $\varepsilon_{n}(\zeta), \zeta^{2} \neq 1$ are simple,

$$
\begin{gathered}
\varepsilon_{n}(\zeta)=\varepsilon_{n}\left(\zeta^{-1}\right), \\
\varepsilon_{2 m-1}(1)<\varepsilon_{2 m}(1), \\
\varepsilon_{2 m}(-1)<\varepsilon_{2 m+1}(-1), \quad m=1,2, \ldots .
\end{gathered}
$$

2) $u_{1}^{+1}$ and $u_{n}^{\zeta}, \zeta^{2} \neq 1$, have no zeros on $J ; u_{n}^{N}$ and $u_{n}^{D}$ have exactly $n-1$ zeros in $(a, b) ; u_{2 m}^{+1}$ and $u_{2 m+1}^{+1}$ have exactly $2 m$ zeros and $u_{2 m-1}^{-1}$ have exactly $2 m-1$ zeros in $[a, b]$ regarded as a cricle, if the respective eigenvalues are nondegenerate. In the case of degeneracy, the statement remains true if the functions are chosen to be real. 3) $\varepsilon_{n}(\zeta)$ are analytic in a neighborhood of $\mathscr{C}=U(1) \backslash\{-1,1\}$ and continuous at $\zeta= \pm 1$; if $\varepsilon_{n}\left(\zeta_{0}\right), \zeta_{0}^{2}=1$ is nondegenerate, then $\varepsilon_{n}$ is analytic at $\zeta_{0}$. When $\zeta$ goes from -1 to +1 on the unit circle $(-1)^{n} \varepsilon_{n}(\zeta)$ increases monotonically.

We refer the reader to Eastham [3], where the proof of most of the assertions may be found.

Theorem 3. (Bracketing of Neumann and Dirichlet eigenvalues). Let $T^{\text {b.c. }}=T_{(a, b)}^{\text {b.c. }}$ and $\varepsilon_{n}^{N(D)}, \varepsilon_{n}(\zeta), n=1,2, \ldots$ be respectively the eigenvalues of $T^{N(D)}$ and $T^{\zeta}$

\footnotetext{
${ }^{2}$ I am indebted to the anonymous referee of this paper who suggested the straightforward proof of Theorem 3 which is given here
} 
respectively, ordered in increasing sequence (if necessary by continuity for $\zeta^{2} \rightarrow 1$ ). Then:

$$
\begin{aligned}
& \varepsilon_{n}\left((-1)^{n}\right) \leq \varepsilon_{n+1}^{N} \leq \varepsilon_{n+1}\left((-1)^{n}\right) ; \\
& \varepsilon_{n}\left((-1)^{n}\right) \leq \varepsilon_{n}^{D} \leq \varepsilon_{n+1}\left((-1)^{n}\right), \quad n=1,2, \ldots ;
\end{aligned}
$$

and all the bounds are attainable.

Proof. Let us first show that neither the Dirichlet nor the Neumann eigenvalues can coincide with any $\varepsilon_{n}(\zeta), \zeta^{2} \neq 1$, i.e. that the Dirichlet/Neumann eigenvalues are either in the gaps or at the band edges.

Assuming the contrary, let $\varepsilon=\varepsilon_{n}(\zeta)$, for some $n \in \mathbb{N}, \zeta^{2} \neq 1$, be a Neumann eigenvalue. By Proposition $1, \varepsilon=\varepsilon_{n}\left(\zeta^{-1}\right)$, so that $u_{n}^{\zeta}$ and $u_{n}^{\zeta^{-1}}$ are linearly independent (and complex conjugate) solutions of the equation:

$$
\frac{-d^{2} u}{d x^{2}}+V u=\varepsilon u \text {. }
$$

Let

$$
u_{n}^{\zeta}(x)=M(x) e^{i \varphi(x)},
$$

with $M>0$ by Proposition 1 . By adding a suitable constant phase to $\varphi$, the (real) Neumann eigenfunction may be written as:

$$
u^{N}(x)=M(x) \cos (\varphi(x)),
$$

while the boundary conditions satisfied by $M$ and $\varphi$ are

$$
\begin{gathered}
M(1)=M(0), \quad M^{\prime}(1)=M^{\prime}(0), \\
\varphi(1)=\varphi(0)+\arg (\zeta)+2 k \pi, \quad \varphi^{\prime}(1)=\varphi^{\prime}(0),
\end{gathered}
$$

for some integer $k$. By assumption, $u^{N}$ satisfies the Neumann conditions, which yield for $M$ and $\varphi$ :

$$
\begin{aligned}
& M^{\prime}(0) \cos (\varphi(0))-\varphi^{\prime}(0) M(0) \sin (\varphi(0))=0, \\
& M^{\prime}(1) \cos (\varphi(1))-\varphi^{\prime}(1) M(1) \sin (\varphi(1))=0 .
\end{aligned}
$$

The compatibility condition of Eqs. (2.8) and (2.9) is

$$
\tan (\varphi(0))=\tan (\varphi(0))+\arg (\zeta))
$$

which implies $\zeta^{2}=1$, contradicting the assumption.

The reasoning in the Dirichlet case is quite similar.

If $V=0$, then

$$
\begin{gathered}
\varepsilon_{n}(\zeta)=\left[(n-1) \pi+(-1)^{n+1}|\arg (\zeta)|\right]^{2}, \\
\varepsilon_{n}^{D}=(n \pi)^{2}, \quad \varepsilon_{n}^{N}=[(n-1) \pi)^{2}, \quad n=1,2, \ldots .
\end{gathered}
$$

The Dirichlet and Neumann eigenvalues satisfy Eq. (2.4) with all the $\leq$ signs replaced by $=$.

Now, for piecewise continuous $V, T^{\text {b.c. }}+g V$ is an entire analytic family (see e.g. [16]). Since all the eigenvalues of the Dirichlet, Neumann and (for $\left.\zeta^{2}=1\right) \zeta$-operators are nondegenerate and for all real $g, \varepsilon_{k}^{N / D}(g) \neq \varepsilon_{k}(\zeta, g), \zeta^{2} \neq 1$, we have

$$
\left.\varepsilon_{n}(g) \zeta\right) \leq \varepsilon_{n}^{D}(g) \leq \varepsilon_{n}^{D}(g) \leq \varepsilon_{n}(\zeta, g), \quad n=1,2, \ldots,
$$

wherefrom Eq. (2.4) follows by the monotonicity in $\zeta$ (Proposition 1, 3). 
It remains to show the attainability of the bounds in Eq. (3.1). Let $V_{\text {per }}$ be the continuation of $V$ to $\mathbb{R}$ by periodicity and define $V_{y}$ on $(a, b)$ by

$$
V_{y}(x)=V_{\text {per }}(x+y), \quad x \in(a, b) .
$$

Let $T_{y}^{\text {b.c. }}(y)=-\Delta^{\text {b.c. }}+V_{y}$ and $\varepsilon_{n}^{\text {b.c. }}(y)$ - its eigenvalues. $T_{y}^{\zeta}$ is unitarily equivalent to $T^{\zeta}=T_{0}^{\zeta}$ by the cyclic translation operator. Since the eigenfunctions of $T^{ \pm 1}$ are real and $C^{1}$, there are points $y_{n, \alpha}^{n, \pm}$ at which $\frac{d}{d x} u_{n}^{ \pm 1}\left(y_{n, \alpha}^{N, \pm}\right)=0$. Remember that $u_{n}^{ \pm 1}$ is (anti) periodic for any $n \in \mathbb{N}$. With the exception of $u_{1}^{+1}$ the eigenfunctions also have zeros: $y_{\eta, \beta}^{D, \pm}$. Thus, the $(n+1)^{\text {st }}$ eigenvalue of $T_{y}^{N}$ attains the lower bound (3.1) for $y=y_{n, \alpha}^{N,(-1)^{n}}$ and the upper one for $y=y_{n+1, \alpha}^{N,(-1)^{n}}$. The Dirichlet case is similar.

Let us now consider $T_{(0, L)}^{\text {b.c. }}, 2 \leq L \in \mathbb{N}$, with a periodic potential, $V(x+1)=V(x)$. It is obvious that the eigenvalues of $T_{(0, L)}^{\zeta}$ may be obtained from those of $T_{(0,1)}^{\zeta}$. By Theorem 3 we may also locate $L-1$ eigenvalues of $T_{(0, L)}^{D(N)}$ in each of the bands of $T$ and bracket the remaining one eigenvalue per gap of $T$. Thus:

Proposition 2. Let $T_{L}^{\text {b.c. }}=T_{(0, L)}^{\text {b.c. }}$, with $V$ having unit period. Let $\varepsilon_{k}(\zeta)$, $u_{k}^{\zeta}(x)$ be the eigenvalues and eigenfunctions of $T_{1}^{\zeta}$ and $\varepsilon_{k}(\zeta, L)$ and $u_{k}^{\zeta}(x, L)$ those for $T_{L}^{\zeta}, L \in \mathbb{N}$, arranged in nondecreasing order. Then

1)

$$
\varepsilon_{(k-1) L+m}(\zeta, L)=\varepsilon_{k}\left(\zeta_{m}^{(L, k)}\right), \quad m=1,2, \ldots, L, k=1,2, \ldots,
$$

where $\zeta_{m}^{(L, k)}$ are the $L$ roots of the equation

$$
\eta^{L}=\zeta
$$

2) The eigenvalues of $T$ satisfy

$$
\begin{gathered}
\varepsilon_{(k-1) L+m}^{D}(L)=\varepsilon_{(k-1) L+m+1}^{N}(L)=\varepsilon_{k}\left((-1)^{k+1} e^{i m \pi / L}\right), \\
\varepsilon_{k}\left((-1)^{k}\right) \leq \varepsilon_{k L}^{D}(L), \quad \varepsilon_{k L+1}^{N}(L) \leq \varepsilon_{k+1}\left((-1)^{k+1}\right),
\end{gathered}
$$

where $m=1,2, \ldots, L-1, k=1,2, \ldots$.

Remark 4. Let $T_{\text {per }}=-\Delta+V_{\text {per }}$ on $L^{2}(\mathbb{R})$. Then, (see e.g. [1])

$$
S p\left(T_{\mathrm{per}}\right)=\bigcup_{\substack{n \in \mathbb{N} \\ \zeta \in U(1)}} \varepsilon_{n}(\zeta)=\bigcup_{n \in \mathbb{N}}\left[a_{n}, b_{n}\right]
$$

where $\alpha_{n}=\varepsilon\left((-1)^{n+1}\right)<b_{n}=\varepsilon_{n}\left((-1)^{n}\right)$.

We have shown that for any $n \in \mathbb{N}$,

$$
\varepsilon_{n+1}^{N}(y), \varepsilon_{n}^{D}(y) \in\left[b_{n}, a_{n+1}\right] .
$$

The periodic functions $\varepsilon_{n+1}^{N}(y)$ and $\varepsilon_{n}^{D}(y)$ oscillate in the interval (2.15) attaining its edges at least once in each period. If the $n^{\text {th }}$ gap is closed, $b_{n}=a_{n+1}$, they are pinned (constant). 


\section{Bracketing Operators without Eigenvalues in a Gap}

As we have seen the Dirichlet and Neumann operators on an interval have generically eigenvalues in the gaps of the $\zeta$ (quasiperiodic) operators. Nevertheless we may use the method of proof of Theorem 3 to construct approximating operators bracketing $H_{\omega}$ which have no eigenvalues in a given gap of $\operatorname{Sp}\left(H_{\omega}\right)$. By using these we will achieve the proof of Theorem 2 in the next section.

By Dirichlet-Neumann bracketing $([5,7,10,12], \sigma$ is bracketed by the expectation values of the integrated density of states for the restrictions of $H_{\omega}$,

$$
L^{-1} \mathbb{E}\left\{\#\left(H_{\omega, L}^{D}-E\right)\right\} \leq \sigma(E) \leq L^{-1} \mathbb{E}\left\{\#\left(H_{\omega, L}^{N}-E\right)\right\},
$$

for any $L \in \mathbb{N}$. As we have seen, for $T_{L}^{N}(g), L-1$ eigenvalues per band are in $S p(T(g))$ and, generically, there is one eigenvalue in each gap of $S p T(g)$. But

Lemma 1. Let $H_{\omega}$ be bounded from above (below) by

$$
H_{\omega}^{\text {b.c. }}(L, y)=\bigoplus_{m \in \mathbb{Z}} H_{\omega,(m L+y,(m+1) L+y)}^{\text {b.c. }},
$$

$L \in \mathbb{N}, y \in\left(-\frac{1}{2}, \frac{1}{2}\right]$, where $H_{\omega,(a, b)}^{\text {b.c. }}$ is the restriction of $H_{\omega}$ to $L^{2}(a, b)$ with boundary conditions b.c. $=D($ b.c. $=N)$. Let $\left(E_{n}^{-}, E_{n}^{+}\right)$be the $n^{\text {th }}$ gap of $H_{\omega}$. Then, one may choose $y=y_{n}^{D}\left(y_{n}^{N}\right)$ such that the IDS of $H_{\omega}^{\text {b.c. }}\left(L, y_{n}^{\text {b.c. }}\right)$

$$
\sigma(E ; L, y)=\sigma(E)=n, \forall E \in\left(E_{n}^{-}, E_{n}^{+}\right) .
$$

Proof. By Proposition 1 there are $L-1$ eigenvalues of $T_{\alpha, \alpha+L}^{D(N)}(g)$ of $T(g)$ in each band of $S p(T(g))$. By Theorem 3 and Remark 4 the eigenvalues that lie generically in the gap are periodic functions of $\alpha$, attaining the spectral edges at least once per period.

Let us consider b.c. $=N$ and choose a $y=y_{n}^{N} \in\left(-\frac{1}{2}, \frac{1}{2}\right]$ for which the $(n+1)^{\text {st }}$ eigenfunction of $T_{1}^{(-1)^{n}}(0)$ has zero derivative. Then, the $(n L+1)^{\text {st }}$ eigenvalue of $T_{\left(y_{n}^{N}, y_{n}^{N}+L\right)}^{N}(0)$

$$
\lambda_{n L+1}\left[T_{\left.y_{n}^{N}, y_{n}^{N}+L\right)}^{N}(0)\right]=\varepsilon_{n+1}\left((-1)^{n}\right)=E_{n}^{+} .
$$

By Proposition 2, for $g=1$

$$
\lambda_{n L}\left[T_{\left(y_{n}^{N}, y_{n}^{N}+L\right)}^{N}(1)\right]=\varepsilon_{n}\left((-1)^{n} e^{\frac{i \pi}{L}}, 1\right)<\varepsilon_{n}\left((-1)^{n}, 1\right)=E_{n}^{-} .
$$

Since

$$
T_{\left(y_{n}^{N}, y_{n}^{N}+L\right)}^{N}(0) \leq H_{\omega,\left(y_{n}^{N}, y_{n}^{N}+L\right)}^{N} \leq T_{\left(y_{n}^{N}, y_{n}^{N}+L\right)}^{N}(1),
$$

we obtain

$$
\lambda_{n L}\left[H_{\omega,\left(y_{n}^{N}, y_{n}^{N}+1\right)}^{N}\right]<E_{n}^{-}
$$

and

$$
\lambda_{n L+1}\left[H_{\omega,\left(y_{n}^{N}, y_{n}^{N}+1\right)}^{N}\right] \geq E_{n}^{+} .
$$

For the Dirichlet case we choose $y=y_{n}^{D} \in\left(-\frac{1}{2}, \frac{1}{2}\right]$ for which the $n^{\text {th }}$ eigenfunction of $T_{1}^{D}(1)$ has a zero. Then, the $n L^{\text {th }}$ eigenvalue of $T_{\left(y_{n}^{D}, y_{n}^{D}+1\right)}^{D}(1)$,

$$
\lambda_{n L}\left[T_{\left(y_{n}^{D}, y_{n}^{D}+L\right)}^{D}\right]=\varepsilon_{n}\left((-1)^{n}, 1\right)=E_{n}^{-},
$$


and using Eq. (3.6) with $N$ replaced by $D$, we obtain

$$
\begin{gathered}
\lambda_{n L}\left[H_{\omega,\left(y_{n}^{D}, y_{n}^{D}+L\right)}^{D}\right] \leq E_{n}^{-}, \\
\lambda_{n L+1}\left[H_{\omega,\left(y_{n}^{D}, y_{n}^{D}+L\right)}^{D}\right]>E_{n}^{+} .
\end{gathered}
$$

It remains to note that Eqs. (3.7), (3.8) and (3.10), (3.11) remain obviously valid if we add an integer to $y$.

\section{Proof of Theorem 2}

In the previous section we have proven Lemma 1 which gives us bracketing operators for $S p\left(H_{\omega}\right)$. Now we can return to our primary task. We will proceed by DirichletNeumann bounding taking a single upper/lower operator for both spectral edges bordering a given gap - the one defined in Lemma 2. For the sake of simpler notations, having set on proving the theorem near the edges $E_{n}^{-}, E_{n}^{+}$of a given gap, we will omit the $n, y, L$ dependence of the bracketing operators, writing $H_{\omega}^{D}$ for $H_{\omega,\left(y_{n}^{D}, y_{n}^{D}+L\right)}^{D}$ and $H_{\omega}^{N}$ in the Neumann case.

Definition 1. Let $X_{ \pm}^{N(D)}(\omega, L, C), C>0$, be the events

$$
\left\{H_{\omega}^{N(D)} \text { has an eigenvalue in the interval }\left(E_{n}^{ \pm}-\frac{C}{L^{2}}, E_{n}^{ \pm}+\frac{C}{L^{2}}\right)\right\} .
$$

The proof becomes a simple exercise given the following:

Lemma 2. For sufficiently large $L$ and $C^{-1}$ there are $L$-independent constants $A, B>0$ such that

$$
\begin{aligned}
& \ln \mathbb{P}\left[X_{ \pm}^{N}(\circ, L, C)\right] \leq-A L \ln L, \\
& \ln \mathbb{P}\left[X_{ \pm}^{D}(\circ, L, C)\right] \geq-B L \ln L,
\end{aligned}
$$

if in Eq. (1.5) $\delta_{ \pm}>0$. For $\delta_{ \pm}=0$ the logarithms should be dropped from the r.h.s. of Eqs. (1-2). Here $\mathbb{P}[X]$ is the probability of the event $X$.

Indeed, $H_{\omega}^{N(D)}$ has no eigenvalues in $\left(E_{n}^{-}, E_{n}^{+}\right)$. For sufficiently small $C>0$, let

$$
S p\left(H_{\omega}\right) \ni E=E_{n}^{ \pm} \pm \frac{C}{L^{2}} .
$$

Taking into account Eqs. (3.7)-(3.11), only $\lambda_{n_{L}}$ may be in $\left[E, E_{n}^{-}\right]$, respectively only $\lambda_{n L+1} \in\left[E_{n}^{+}, E\right]$. Thus, by Eq. (4.1), $\sigma(E)-\sigma\left(E_{n}^{+}\right)$is bracketed by $f_{D}(E), f_{N}(E)$, with

$$
f_{D / N}(E)=\frac{1}{L(E)} \mathbb{P}\left[X_{+}^{D / N}(\circ, L(E), C)\right]
$$

$L(E)=C\left|E-E_{n}^{+}\right|^{-1 / 2}$ and a similar pair for $\sigma\left(E_{n}^{-}\right)-\sigma(E)$. Taking the limit $E \rightarrow E_{n}^{ \pm}$yields Eq. (1.17).

Before proceeding further we will state a generalization by Kirsch and Nitzschner [6] of Temple's inequality, which may be proven in the same way as Theorem XIII.5 of [16].

Lemma 3. Let $H$ be selfadjoint, semibounded and with compact resolvent. Let 
Lemma 3. Let $H$ be selfadjoint, semibounded and with compact resolvent. Let

$$
\lambda_{n}(H) \leq \nu_{n}<0<\nu_{n+1} \leq \lambda_{n+1}(H),
$$

and $\varphi \in \mathscr{D}(H),\|\varphi\|=1,(\varphi, H \varphi)=0$. Then:

$$
\begin{aligned}
\lambda_{n}(H) & \geq-\frac{\|H \varphi\|^{2}}{\nu_{n+1}} \\
\lambda_{n+1}(H) & \leq-\frac{\|H \varphi\|^{2}}{\nu_{n}} .
\end{aligned}
$$

Proof of Lemma 2. Let us start with Eq. (1) for $E_{n}^{-}$. For $-1 \leq i \leq L+1$ and some $\xi \in[0,1]$ define

$$
q_{i}\left(\omega_{N}\right)= \begin{cases}1-\xi, & \text { if all } q_{j}(\omega) \geq 1-\xi,-1 \leq j \leq L+1 \\ 0, & \text { otherwise }\end{cases}
$$

Obviously,

$$
H_{\omega_{N}}^{N} \leq H_{\omega}^{N}
$$

so that $X_{-}^{N}\left(\omega_{N}, L, C\right) \Rightarrow X_{-}^{N}(\omega, L, C)$. But

$$
H_{\omega_{N}}^{N}=T^{N}(1-\xi)
$$

in the first case in Eq. (7) and $H_{\omega_{N}}=T^{N}(0)$ in the second. In the latter case there are no eigenvalues in $\left(E_{n}^{-}-\frac{C}{L^{2}}, E_{n}^{-}\right)$for sufficiently small $C L^{-2}$. In the former, by Proposition 2 and Theorem 3 for $g=1-\xi$,

$$
\lambda_{n L}\left(T^{N}(1-\xi)\right)=\varepsilon_{n}\left(e^{i \pi(n-L-1)}, 1-\xi\right) .
$$

Since $\varepsilon_{n}\left((-1)^{n}, g\right)$ is not degenerate for $g$ in some neighborhood of 1 , it will be analytic in $\zeta$ near $\zeta_{0}=(-1)^{n}$ and also analytic in $g$ near $g=1$. For sufficiently small $L^{-1}$ and $\zeta$,

$$
\varepsilon_{n}\left((-1)^{n} e^{\frac{2 \pi}{L}}, 1-\xi\right)=\varepsilon_{n}\left((-1)^{n}, 1\right)-\frac{\alpha}{L^{2}}-\left.\xi \frac{\partial \varepsilon_{n}\left((-1)^{n}, q\right)}{\partial g}\right|_{g=1-\xi}+\ldots
$$

Taking $\xi=\frac{\beta}{L^{2}}$, noting that $\frac{\partial \varepsilon_{n}(\zeta, g)}{\partial g}>0$, and using Eq. (1.5), we see that, if $\delta_{-}>0$, Eq. (1.1) is valid while for $\delta_{-}=0$ there is no $\ln L$ term.

To prove (4.2) near $E_{n}^{-}$, let us redefine again $q_{i}(\omega)$ by

$$
q_{i}\left(\omega_{D}\right)= \begin{cases}1, & \text { if } q_{i}(\omega)>1-\xi \\ 1-\xi, & \text { if } q_{i}(\omega) \leq 1-\xi\end{cases}
$$

for some $\xi \in[0,1],-1 \leq i \leq L+1$. Since

$$
H_{\omega_{D}}^{D} \leq H_{\omega}^{D}
$$

then $X_{-}^{D}(\omega, L, C) \Rightarrow X_{-}^{D}\left(\omega_{D}, L, C\right)$. Now,

$$
H_{\omega_{D}}^{D}=T^{D}(1)-\sum_{i}\left[1-q_{i}\left(\omega_{D}\right)\right] f(\circ-i) .
$$


Let

$$
\varphi=\frac{1}{L} u_{n}^{(-1)^{n}}, \quad\|\varphi\|=1
$$

where $u_{n}^{(-1)^{n}}$ is the normalized $n^{\text {th }}$ eigenfunction of $T_{1}^{D}(1)$ continued periodically and restricted to our interval:

$$
T^{D}(1) \varphi=\lambda_{n L}\left[T^{D}(1)\right] \varphi .
$$

Since the sum in Eq. (5.13) is nonnegative,

$$
\left.\lambda_{n L-1}\left[H_{\omega_{D}}^{D}\right] \leq \varepsilon_{n}\left((-1)^{n}\right) e^{\frac{2 \pi}{L}}, 1\right) \leq E_{n}^{-}-\frac{\alpha}{L^{2}},
$$

for $L$ sufficient large, where we used Proposition 2. Now

$$
\left(\varphi, H_{\omega_{D}}^{D} \varphi\right)=E_{n}^{-}-\frac{N_{+} h_{1} \beta}{L^{3}}=F
$$
where $N_{+}$is the number of $q_{i}\left(\omega_{D}\right)$ which are $=1-\zeta, h_{1}=\int_{0}^{1} f(x)\left|u_{n}^{(-1)^{n}}(x)\right|^{2} d x>0$
and we set $\zeta=\beta L^{-2}$. Defining

$$
H=H_{\omega_{D}}^{D}-F,
$$

and choosing $\beta<\alpha / h_{1}$ we may apply Lemma 4 to obtain an upper bound to $\lambda_{n_{L}}\left(H_{\omega_{D}}^{D}\right)$ :

$$
\lambda_{n L}\left(H_{\omega_{D}}^{D} \leq E_{n}^{-}-\frac{N_{+} \beta h_{1}}{L^{3}}+\frac{N_{+} \beta^{2} h_{2}^{2}}{L^{3}} \frac{1-N_{+} h_{1}^{2} / L h_{2}^{2}}{\alpha-N_{+} h_{1} \beta / L} .\right.
$$

Here $h_{2}^{2}=\int_{0}^{1} f^{2}(x)\left|u_{n}^{(-1)^{n}}(x)\right|^{2} d x$. Now by standard large derivation arguments (see e.g. Sect. 4 of [11]) we may establish Eq. (4.2) for $E_{n}^{-}$. The case of $E_{n}^{+}$is essentially the same.

Acknowledgements. I thank Sergio Albeverio for hospitality at the Institut für Mathematik of the Ruhr-Universität Bochum and SFB 237, Unordnung und Große Fluktuationen, for financial support. Discussions with Roger Balian, Anton Bovier, Jean-Marc Luck and Frank Nitzschner are gratefully acknowledged. Special thanks are due to Werner Kirsch for many discussions and stimulating suggestions.

\section{References}

1. Carmona, R., Lacroix, J.: Spectral theory of Random Schrödinger operators. Boston, Basel, Berlin: Birkhäuser 1990

2. Eastham, M.S.P.: The spectral theory of periodic differential equations. Edinburgh: Scottish Academic Press 1983

3. Fukushima, M.: On the spectral distribution of a disordered system and the range of a random walk. Osaka J. Math. 11, 73-85 (1974)

4. Kirsch, W., Martinelli, F.: On the spectrum of Schrödinger operators with a random potential. Commun. Math. Phys. 85, 329-350 (1985)

5. Kirsch, W., Martinelli, F.: Large deviations and the Lifschitz singularity of the integrated density of states of random Hamiltonians. Commun. Math. Phys. 89, 27-40 (1983) 
6. Kirsch, W., Nitzschner, F.: Lifschitz tails and non-Lifschitz tails for one dimensional random point interactions. Operator theory: advanced applications, Vol. 46. Exner, P., Neidhardt, H. (eds.) Basel: Birkhäuser 1990

7 Kirsch, W., Simon, B.: Lifschitz tails for periodic plus random potentials. J. Stat. Phys. 42, 799-808 (1986)

8. Lifschitz, I.M.: The energy spectrum of disordered systems. Adv. Phys. 13, 483-536 (1964)

9. Lifschitz, I.M., Gredeskul, S.A., Pastur, L.A.: Introduction to the theory of disordered systems. Moscow: Nauka 1982

10. Mezincescu, G.A.: Bounds on the integrated density of electronic states for disordered Hamiltonians. Phys. Rev. B 32, 6272 (1985)

11. Mezincescu, G.A.: Internal Lifschitz singularities of disordered finite-difference Schrödinger operators. Commun. Math. Phys. 103, 167-176 (1986)

12. Mezincescu, G.A.: Lifschitz singularities for periodic plus random potentials. J. Stat. Phys. 49, $1181-1190(1987)$

13. Pastur, L.A.: On the distribution of eigenvalues of the Schrödinger equation with a random potential. Funkt. Anal. Appl. 6, 163-165 (1972)

14. Pastur, L.A.: Behaviour of some Wiener integrals as $t \rightarrow \infty$ and the density of states of Schrödinger's equation with a random potential. Teor. Mat. Fiz. 32, 88-95 (1977)

15. Pastur, L.A.: Spectral properties of disordered systems in the one-body approximation. Commun. Math. Phys. 75, 179-196 (1980)

16. Reed, M., Simon, B.: Methods of modern mathematical physics. IV. Analysis of operators. New York: Academic 1978

17. Simon, B.: Lifschitz tails for the Anderson model. J. Stat. Phys. 38, 65-76 (1985)

18. Simon, B.: Internal Lifschitz tails. J. Stat. Phys. 46, 911-918 (1987)

19. Simon, B.: Regularity of the density of states for stochastic Jacobian matrices. A review. In: Random media. Papanicolaou, G. (ed.) New York: Springer 1987, pp. 245-266

Communicated by B. Simon 
